\title{
Experiencia en nefrolitotomía percutánea modificada en un centro urológico
}

\section{Experience in modified percutaneous nephrolithotomy in a urology centre}

Jaime Velasco-Piedrahita, $\mathrm{MD}^{1} \quad$ Andrés Gaviria-Mendoza, $\mathrm{MD}^{2} \quad$ Jose William Martínez, $\mathrm{MD}^{3}$

1 Urólogo, Calculaser S.A., Pereira, Risaralda, Colombia

2 Universidad Tecnológica de Pereira, Pereira, Risaralda, Colombia

${ }^{3}$ Epidemiólogo, Universidad Tecnológica de Pereira, Pereira,

Risaralda, Colombia

Urol Colomb 2018;27:81-85.
Address for correspondence Jaime Velasco-Piedrahita, Urólogo, Calculaser S.A., Pereira, Risaralda, Colombia

(e-mail: javepala@gmail.com).

\section{Resumen}

Palabras clave

- atención ambulatoria

- nefrolitiasis

- nefrolitotomía percutánea

- nefrostomía percutánea

- tubeless
Introducción Recientemente se ha descrito la técnica quirúrgica para la realización de nefrolitotomías percutáneas modificadas que permite obtener punciones de diámetro reducido, con buena visibilidad y éxito sin la necesidad de adquirir nuevos instrumentos.

Objetivo Establecer el comportamiento de los pacientes tratados con nefrolitotomía percutánea modificada en un centro urológico de Pereira (Colombia), 2015-2016.

Métodos Estudio observacional descriptivo de un grupo de pacientes que han sido tratados con nefrolitotomía percutánea modificada, donde se eliminó la camisa metálica del nefroscopio estándar para permitir menor diámetro del tracto de entrada (22-F). Se revisaron datos de registros clínicos incluyendo variables sociodemográficas, de comorbilidades, quirúrgicas y complicaciones. Se realizó seguimiento de variables adicionales del postoperatorio. Estadística descriptiva empleando STATA 11.

Resultados En total se revisaron datos de 21 pacientes intervenidos con nefrolitotomía percutánea modificada. La edad media fue de 51,5 años y el 57,1\% fueron hombres. El tamaño promedio de las litiasis fue de $32,8 \mathrm{~mm}$ y el $57,1 \%$ de las punciones fueron en cáliz inferior. La duración promedio del procedimiento fue de 91 min con una mediana de sangrado de $50 \mathrm{~mL}$. La tasa libre de cálculos fue del $90,5 \%$ y ningún paciente tuvo sonda de nefrostomía (tubeless). La mayoría de los pacientes (95,2\%) fueron manejados de manera ambulatoria, sin mayores complicaciones intra- o posquirúrgicas, aunque el nivel de dolor a la semana siguiente del procedimiento fue en promedio de 4,1 .

Conclusiones La técnica de nefrolitotomía percutánea modificada descrita mostró ser segura, efectiva y factible de realizar, incluso en pacientes con grandes litiasis y tubeless ambulatoria. received

October 24, 2016

accepted

March 25, 2017

published online

January 11, 2018
DOI https://doi.org/

10.1016/j.uroco.2017.03.014.

ISSN 0120-789X.

eISSN 2027-0119.
Copyright ( 2018, Sociedad Colombiana License terms de Urología. Publicado por Thieme Revinter Publicações Ltda., Rio de Janeiro, Brazil. Todos los derechos reservados.

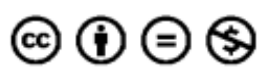




\begin{abstract}
Introduction A surgical technique has been recently described for performing modified percutaneous nephrolithotomy that enables small diameter punctures to be made, with good visibility and success and without the need to purchase new instruments.

Objective To establish the outcomes of patients treated with modified percutaneous nephrolithotomy in a Urology Centre in Pereira (Colombia), 2015-2016.

Methods Observational study of a group of patients who were treated with modified percutaneous nephrolithotomy, where the outer sheath of the standard nephroscope was removed to give a smaller tract diameter (22-F). The data from clinical records were recorded, including socio-demographics, comorbidities, and surgical and other complications. Additional post-operative variables were monitored during follow-up. Descriptive statistics were performed using STATA 11.

Results The data from a total of 21 patients who underwent modified percutaneous nephrolithotomy were reviewed. The mean age was 51.5 years, and $57.1 \%$ were men. The

\section{Keywords}

- ambulatory care

- nephrolithiasis

- percutaneous nephrostomy

- percutaneous nephrolithotomy

- tubeless mean size of the stones was $32.8 \mathrm{~mm}$, and $57.1 \%$ of punctures were in lower calyx. The mean duration of the procedure was 91 minutes, with a median bleeding of $50 \mathrm{~mL}$. The stone-free rate was $90.5 \%$, and no patient had a nephrostomy tube (tubeless). Most patients (95.2\%) were managed on an outpatient basis, without major intra- or post-operative complications, although the mean pain level was 4.1 in the week following the procedure. Conclusions The modified percutaneous nephrolithotomy technique showed to be safe, effective, and feasible to perform, as well as tubeless in ambulatory patients and with large stones.
\end{abstract}

\section{Introducción}

La nefrolitotomía percutánea es una técnica quirúrgica empleada en urología desde 1970 como terapia de primera línea para el manejo de litiasis renales de gran tamaño, múltiples o las ubicadas en el cáliz inferior. ${ }^{1}$ Desde la primera vez que se empleó, la técnica ha venido evolucionando para hacerse más efectiva y segura, especialmente al compararla con técnicas abiertas. $^{2}$ Uno de los grandes cambios fue la realización de la cirugía sin dejar tubos de nefrostomía luego de la intervención (tubeless en inglés), lo que se ha asociado con menor dolor posquirúrgico, menor consumo de analgésicos, disminución en el tiempo de recuperación ${ }^{2,3}$ y estancias hospitalarias más cortas. ${ }^{4}$

Recientemente se han creado instrumentos que permiten la realización de este tipo de cirugías con menor daño a los tejidos al emplear nefroscopios y materiales de desintegración de cálculos de diámetro reducido, generando las técnicas conocidas como minipercutánea, ultraminipercutánea y micropercutánea, las cuales son seguras y efectivas en manejar litiasis desde pequeñas hasta moderado tamaño. ${ }^{5-7}$ Sin embargo, estas técnicas han presentado algunos inconvenientes derivados precisamente del uso de instrumentos más pequeños, como son menor visibilidad e irrigación durante la intervención así como tiempos quirúrgicos más prolongados, ${ }^{7,8}$ además de que al emplear nuevas herramientas se incrementan los costos de operación, lo cual puede ser un inconveniente mayor en sistemas de salud que cuentan con escasos recursos.

Se ha descrito que al comparar grupos de pacientes en los cuales se empleó una camisa de Amplatz de menor diámetro contra las convencionales, se reducía la cantidad de hemorragia y daño en la función renal. ${ }^{9}$ Lipsky et al. describieron en 2013 una técnica de nefrolitotomía percutánea modificada en la cual emplearon los equipos usados de manera rutinaria pero quitaron la camisa de metal del nefroscopio de 26-F, evitando perder visibilidad e irrigación a pesar de haber generado tractos de acceso de menor diámetro similares a la minipercutánea, logrando una elevada tasa de éxito. ${ }^{7}$ Asimismo, indican que de preferencia esta técnica debe ser tubeless, ${ }^{7}$ lo que podría representar menor morbilidad y mayor costo-efectividad. ${ }^{10}$

Teniendo en cuenta las bondades de las técnicas quirúrgicas donde se emplean instrumentos de diámetro reducido y que con una simple modificación del material quirúrgico estándar se pueden realizar percutáneas menos invasivas sin sobrecostos, el equipo quirúrgico de un centro urológico especializado realizó la técnica modificada de Lipsky et al. en un grupo de pacientes atendidos por litiasis renal. El presente estudio descriptivo muestra las variables clínicas y quirúrgicas de estos pacientes, lo cual puede ser un punto de partida para mejorar la atención de personas con esta enfermedad sin generar mayores gastos económicos al sistema de salud.

\section{Metodología}

Estudio observacional descriptivo de una serie clínica conformada por pacientes con urolitiasis, de un centro especializado, intervenidos con nefrolitotomía percutánea modificada basados en el método descrito por Lipsky et al. ${ }^{7}$ 
El muestreo fue por conveniencia, seleccionándose aquellos atendidos entre mayo de 2015 y mayo de 2016.

Las variables clínicas, quirúrgicas y demográficas se obtuvieron de la base de datos institucional y se complementaron con entrevistas telefónicas (realizadas por personal de salud del centro o por los investigadores), incluyendo:

- Género, edad, lugar de residencia.

- Comorbilidades: hipertensión arterial, diabetes, hipotiroidismo, obesidad.

- Tipo de litiasis y su tamaño: coraliforme completo vs. otros tipos de cálculos incluyendo pélvicos, múltiples o del cáliz.

- Presencia de anormalidades anatómicas renales.

- Quirúrgicas: tipo de anestesia, tiempo quirúrgico, tipo de decúbito (supino/prono), lateralidad del riñón intervenido, punción del cáliz (inferior, medio, superior o múltiples), litiasis residual (cálculos mayores de $3 \mathrm{~mm}$ ), tubo de nefrostomía (sí/ no), tiempo de estancia en observación, dolor posquirúrgico inmediato y a la semana siguiente del procedimiento (escala verbal análoga, intensidad de 0 [sin dolor] a 10 [máximo]), complicaciones (sangrado, transfusiones, daño a órganos abdominales, sepsis, peritonitis, muerte).

- Alteraciones posquirúrgicas hasta una semana después de la intervención: infecciones de sitio operatorio, hematuria, fiebre, retención urinaria/incontinencia urinaria, consulta al servicio de urgencias durante la semana siguiente al procedimiento, motivo de consulta a urgencias y la necesidad de hospitalización posterior a esa consulta.

La información se consolidó a través de Microsoft Excel 2007 y el análisis univariado se realizó con el paquete estadístico STATA 11 (StataCorp. 2009. College Station, TX, EE. UU.).

\section{Aspectos bioéticos}

Este proyecto fue avalado por el Comité de Bioética de la Universidad Tecnológica de Pereira en la categoría de investigación sin riesgo según la Resolución 8430 de 1993 donde se establecen los parámetros científicos, técnicos y administrativos para la investigación en salud de Colombia.

\section{Resultados}

Se realizaron en total 25 procedimientos durante el tiempo de estudio, aunque solo se logró completar el seguimiento a 21 pacientes. La edad media fue de $51,5 \pm 14,4$ años (rango: 30 a 85 años). El 57,1\% fueron hombres ( $n=12$ ) y el $61,9 \%$ residía en la ciudad de Pereira. En cuanto a comorbilidades, el $28,6 \%(n=6)$ tenían historia de hipertensión arterial, el $14,3 \%(\mathrm{n}=3)$ diabetes mellitus tipo 2 ; había 2 pacientes con insuficiencia renal crónica y 2 con hipotiroidismo. El $33,3 \%(n=7)$ sufrían de otras afecciones, incluyendo antecedente de infarto, insuficiencia cardíaca o tabaquismo.

En promedio, el índice de masa corporal fue de $27,6 \pm 5,3 \mathrm{~kg} / \mathrm{m}^{2}$, encontrando 6 pacientes (28,6\%) con obesidad (índice de masa corporal $>30$ ), incluso un paciente con índice de masa corporal $=44,4 \mathrm{~kg} / \mathrm{m}^{2}$.
La mayoría de los pacientes fueron intervenidos por litiasis ubicadas en riñón derecho $(61,9 \%, \mathrm{n}=13)$. Un total de 10 pacientes $(47,6 \%)$ presentaban cálculos coraliformes y el diámetro promedio de las litiasis fue de 32,8 $\pm 11,8 \mathrm{~mm}$.

Adicionalmente se lograron identificar 3 pacientes con alguna anormalidad anatómica (estenosis pieloureteral, doble sistema colector, pelvis bífida)

\section{Variables quirúrgicas}

Todas las cirugías fueron guiadas empleando fluoroscopio de arco en $C$, inicialmente insertando catéter ureteral en el lado afectado para tomar imágenes contrastadas.

Luego de acceder al colector con aguja $18-\mathrm{G}$, se pasó una guía 0,38-F y se realizó dilatación del tracto con un set Alken hasta 22-F. Se empleó una camisa Amplatz de 22-F. Se utilizó nefroscopio rígido 26-F (Karl Storz, Alemania) al cual se le retiró la camisa metálica externa, permitiendo el paso de la lente por el tracto de menor diámetro (Amplatz -22F) (-Fig. 1). La fragmentación de los cálculos se logró de manera tradicional con litotriptor neumático (Lithoclast ${ }^{\mathrm{TM}}$ ) y la extracción de los fragmentos con pinza caimán (Karl Storz, Alemania).

En todos los procedimientos se empleó anestesia general y fueron realizados en prono. Ningún paciente tuvo sonda de nefrostomía (tubeless) y solamente 3 fueron dejados también sin catéter doble J (totally tubeless). No se realizó hemostasia coagular o con gel del sitio de punción.

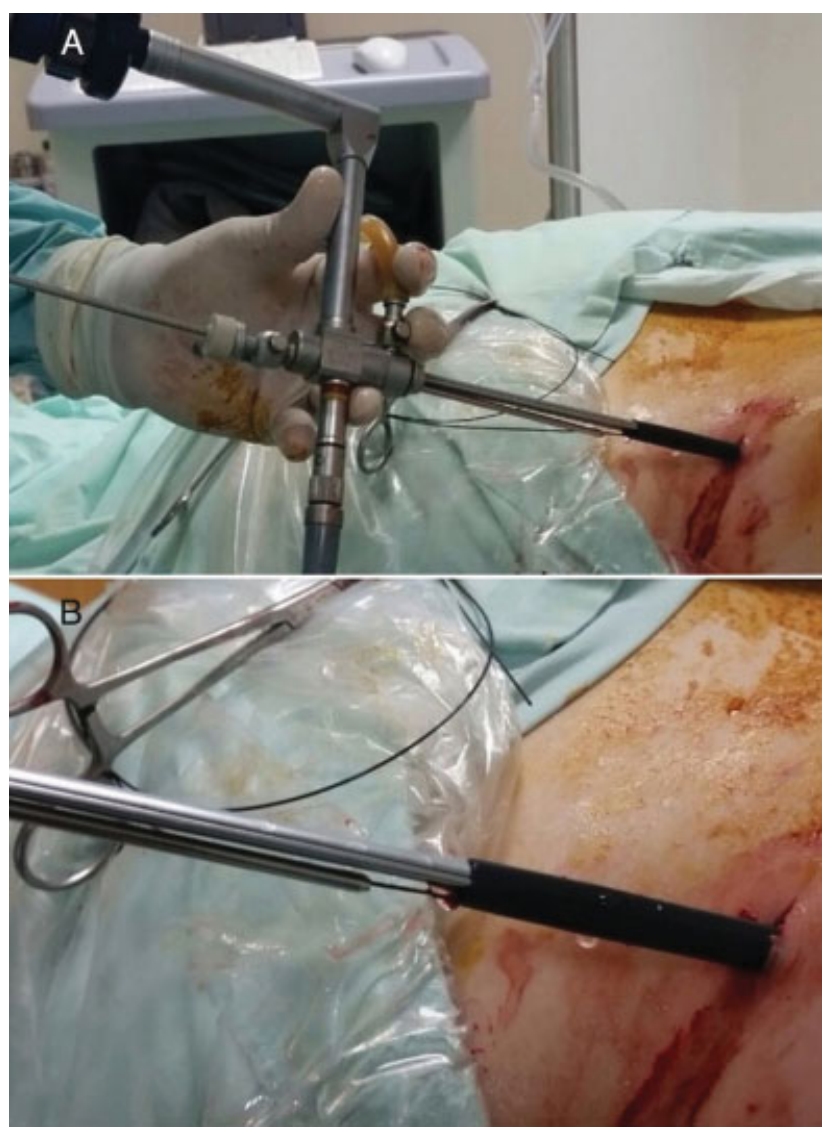

Fig. 1 A. Nefroscopio rígido 26-F al cual se le ha retirado la camisa. B. Paso de nefroscopio sin camisa a través de tracto percutáneo. 
Las punciones fueron en su mayoría realizadas hacia el cáliz inferior ( $\mathrm{n}=12 ; 57,1 \%$, solas o en combinación con una segunda punción), seguidas del superior $(n=7)$ y medio $(\mathrm{n}=6)$. Dos pacientes requirieron 2 punciones en el mismo procedimiento y solamente un paciente necesitó 3 .

La duración promedio del procedimiento fue de $91 \pm 26$ min (rango: 59-154 min) con una mediana de sangrado quirúrgico estimado de $50 \mathrm{~mL}$ (116 mL en promedio). Ningún paciente requirió transfusión sanguínea.

Solamente 5 pacientes $(23,8 \%)$ presentaron litiasis residual al finalizar el procedimiento, con un diámetro estimado promedio de 5,6 $\pm 2,3 \mathrm{~mm}$.

Tres de ellos lograron expulsar el cálculo durante el período de seguimiento (alcanzando una tasa libre de cálculos del 90,5\% a la semana siguiente del procedimiento, según revisión de radiografías simples de abdomen y tomografía computarizada [uro-TAC] de control), mientras que los 2 restantes necesitaron una reintervención con litotricia extracorpórea.

Ningún procedimiento tuvo que ser suspendido por complicaciones intraoperatorias. Solamente se envió un paciente a hospitalización mientras que los demás fueron observados mínimo durante $6 \mathrm{~h}$ antes del alta, garantizando en este momento un control total del dolor posquirúrgico (manejo usual con diclofenaco intramuscular [75 mg/24 h por 3 días] y N-butilbromuro de hioscina).

\section{Variables de seguimiento}

En promedio los pacientes reportaron un nivel de dolor en la escala verbal análoga de 4,1 $\pm 3,2$ a la semana siguiente al procedimiento.

Un paciente manifestó incontinencia urinaria durante este período mientras que 5 referían continuar con hematuria.

Dos pacientes consultaron a urgencias luego del alta. Uno fue diagnosticado con una infección de vías urinarias, aunque no fue hospitalizado. El segundo consultó por hematuria y decidieron hospitalizar según sus comorbilidades (paciente de 85 años hipertenso con falla renal).

Ningún paciente reportó fiebre o infección del sitio quirúrgico hasta una semana después de la intervención. No se presentaron desenlaces fatales.

\section{Discusión}

Las técnicas endourológicas han avanzado considerablemente en los últimos años y la nefrolitotomía percutánea no ha sido la excepción, pasando por diferentes modificaciones que buscan brindar mejores resultados a los pacientes, como son las técnicas tubeless o minipercutáneas.

En el presente trabajo se describe el manejo exitoso de litiasis renal a través de nefrolitotomía percutánea modificada, con un menor diámetro del tracto de entrada sin necesidad de instrumental especial adicional.

La edad promedio de los pacientes es similar a la descrita en otras series, 2,11 incluyendo reportes propios previos con nefrolitotomía percutánea estándar, ${ }^{12}$ aunque las técnicas conocidas como minipercutáneas usualmente se emplean también en población pediátrica. ${ }^{13}$ Las comorbilidades igualmente fueron comparables con datos anteriores, siendo la hipertensión arterial y la diabetes mellitus las más prevalentes. $^{12}$

En cuanto a las variables quirúrgicas, el tiempo de duración del procedimiento, $91 \mathrm{~min}$, fue menor al descrito por Lipsky et al. (116 min), ${ }^{7}$ aunque es superior a la técnica estándar, como se ha descrito previamente. ${ }^{14}$ Por otro lado, el tamaño de las litiasis en promedio fue muy superior al valor de comparación (32,8 vs. $19,4 \mathrm{~mm}){ }^{7}$ pero a pesar de esto se alcanzó un buen porcentaje de tasa libre de cálculos. El sangrado fue menor al compararse con el procedimiento sin modificación ${ }^{12}$ y no se necesitaron transfusiones, lo cual es esperado por ser una técnica con menor daño a tejido. ${ }^{14}$

El dolor en el posquirúrgico inmediato y al momento del alta se pudo controlar exitosamente gracias al cercano cuidado brindado a los pacientes, sin embargo se documentó, según la escala verbal, un promedio de dolor de 4,1 a la semana siguiente al procedimiento. Se esperaría un menor nivel de dolor teniendo en cuenta el diámetro reducido de acceso y que no se dejó nefrostomía, ${ }^{11,14}$ aunque las molestias se podrían explicar por el tubo doble $\mathrm{J}$ y porque las litiasis intervenidas fueron grandes y muchas coraliformes.

Se debe resaltar que todos los procedimientos fueron tubeless (incluso 3 totally tubeless), contrario a la serie de Lipsky et al. donde solamente ocurrió en el $10 \%$ de los pacientes. ${ }^{7}$ Este tipo de técnicas han sido estudiadas previamente, demostrando su seguridad, incluso en pacientes geriátricos. ${ }^{3,4,11,15}$

Otro aspecto relevante es que solamente un paciente fue hospitalizado luego de la intervención, mostrando que la técnica tiene desenlaces positivos con manejo ambulatorio. Además no se presentaron complicaciones intraoperatorias y las consultas a urgencias en el posquirúrgico fueron pocas y de baja gravedad.

Las limitaciones de este trabajo incluyen su técnica retrospectiva de recolección de información y el tiempo restringido de seguimiento luego de la intervención. Además, no se dispone de los datos precisos del manejo del dolor, lo cual es de interés debido al nivel de dolor posquirúrgico no controlado reportado por los pacientes, configurando un punto de interés a intervenir en el centro urológico especializado donde se realizaron las nefrolitotomías.

Aunque se requieren nuevos estudios que comparen prospectivamente sus desenlaces respecto a las técnicas tradicionales o con instrumental especializado, ${ }^{7}$ consideramos que la técnica de nefrolitotomía percutánea modificada es segura y factible de realizar en los pacientes con grandes litiasis, incluso con procedimientos tubeless y de manera ambulatoria, y que además no representa costos adicionales en equipos. Se debe considerar además la experticia del cirujano, quien debe contar con una curva de aprendizaje prolongada en nefrolitotomía percutánea y un adecuado manejo del instrumental para evitar problemas al emplear el nefroscopio sin camisa (posibles complicaciones o deterioro del lente).

\section{Responsabilidades éticas}

\section{Protección de personas y animales}

Los autores declaran que para esta investigación no se han realizado experimentos en seres humanos ni en animales. 


\section{Confidencialidad de los datos}

Los autores declaran que han seguido los protocolos de su centro de trabajo sobre la publicación de datos de pacientes.

\section{Derecho a la privacidad y consentimiento informado} Los autores declaran que en este artículo no aparecen datos de pacientes.

\section{Financiación}

Este trabajo recibió financiación de Calculaser S.A y la Universidad Tecnológica de Pereira.

\section{Conflicto de intereses}

Los autores declaran no tener ningún conflicto de intereses.

\section{Bibliografía}

1 Michel MS, Trojan L, Rassweiler JJ. Complications in percutaneous nephrolithotomy. Eur Urol 2007;51:899-906

2 Shahrour W, Andonian S. Ambulatory percutaneous nephrolithotomy: Initial series. Urology 2010;76:1288-1292

3 Amer T, Ahmed K, Bultitude M, Khan S, Kumar P, de Rosa A, et al. Standard versus tubeless percutaneous nephrolithotomy: A systematic review. Urol Int 2012;88:373-382

4 Zhong Q, Zheng C, Mo J, Piao Y, Zhou Y, Jiang Q. Total tubeless versus standard percutaneous nephrolithotomy: A meta-analysis. J Endourol 2013;27:420-426

5 Armagan A, Tepeler A, Silay MS, Ersoz C, Akcay M, Akman T, et al. Micropercutaneous nephrolithotomy in the treatment of moderate-size renal calculi. J Endourol 2013;27:177-181
6 Tepeler A, Armagan A, Sancaktutar AA, Silay MS, Penbegul N, Akman $T$, et al. The role of microperc in the treatment of symptomatic lower pole renal calculi. J Endourol 2013;27:13-18

7 Lipsky MJ, Shapiro EY, Cha DY, Gupta M. Modified-PCNL without modified instruments: A description of technique. J Endourol 2013;27:684-687

8 Mishra S, Sharma R, Garg C, Kurien A, Sabnis R, Desai M. Prospective comparative study of miniperc and standard PNL for treatment of 1 to $2 \mathrm{~cm}$ size renal stone. BJU Int 2011; 108:896-899

9 Karakose A, Aydogdu O, Atesci YZ. Does the use of smaller Amplatz sheath size reduce complication rates in percutaneous nephrolithotomy? Urol J 2014;11:1752-1756

10 Feng MI, Tamaddon K, Mikhail A, Kaptein JS, Bellman GC. Prospective randomized study of various techniques of percutaneous nephrolithotomy. Urology 2001;58:345-350

11 Álvarez Villarraga JD, Carreño Galeano GL, Hernández García CE, Silva Herrera JM, Patiño Sandoval GA. Nefrolitotomía percutánea convencional vs. tubeless. ¿Es realmente necesaria la derivación urinaria? Urol Colomb 2016;25:5-9

12 Velasco J, Muñoz A, Romero V, Botia N, Gaviria A, Martínez JW. Experiencia en nefrolitotomía percutánea con manejo ambulatorio vs hospitalización en un centro urológico, Pereira, 2009-2012. Urol Colomb 2014;23:165-170

13 Ferakis N, Stavropoulos M. Mini percutaneous nephrolithotomy in the treatment of renal and upper ureteral stones: Lessons learned from a review of the literature. Urol Ann 2015; 7:141-148

14 Zhu W, Liu Y, Liu L, Lei M, Yuan J, Wan SP, et al. Minimally invasive versus standard percutaneous nephrolithotomy: A meta-analysis. Urolithiasis 2015;43:563-570

15 Kara C, Resorlu B, Bayindir M, Unsal A. A randomized comparison of totally tubeless and standard percutaneous nephrolithotomy in elderly patients. Urology 2010;76:289-293 